\title{
Activation of the latent PlcR regulon in Bacillus anthracis

\begin{abstract}
Correspondence
Stephen H. Leppla
\end{abstract} \\ sleppla@niaid.nih.gov \\ Received 10 May 2010 \\ Revised 28 July 2010 \\ Accepted 29 July 2010

\author{
Inka Sastalla, ${ }^{1}$ Lauren M. Maltese, ${ }^{1}$ Olga M. Pomerantseva, ${ }^{2} \ddagger$ \\ ${ }^{1}$ Laboratory of Bacterial Diseases, National Institute of Allergy and Infectious Diseases, \\ National Institutes of Health, Bethesda, MD, USA \\ ${ }^{2}$ Biological Defense Research Directorate, Naval Medical Research Center, Rockville, MD, USA
} Andrei P. Pomerantsev, ${ }^{1}$ Andrea Keane-Myers ${ }^{2}$ and Stephen H. Leppla ${ }^{1}$

\begin{abstract}
Many genes in Bacillus cereus and Bacillus thuringiensis are under the control of the transcriptional regulator PlcR and its regulatory peptide, PapR. In Bacillus anthracis, the causative agent of anthrax, PlcR is inactivated by truncation, and consequently genes having PlcR binding sites are expressed at very low levels when compared with $B$. cereus. We found that activation of the PlcR regulon in $B$. anthracis by expression of a PlcR-PapR fusion protein does not alter sporulation in strains containing the virulence plasmid pXO1 and thereby the global regulator AtxA. Using comparative 2D gel electrophoresis, we showed that activation of the PlcR regulon in $B$. anthracis leads to upregulation of many proteins found in the secretome of $B$. cereus, including phospholipases and proteases, such as the putative protease BA1995. Transcriptional analysis demonstrated expression of BA1995 to be dependent on PlcR-PapR, even though the putative PlcR recognition site of the BA1995 gene does not exactly match the PlcR consensus sequence, explaining why this protein had escaped recognition as belonging to the PlcR regulon.

Additionally, while transcription of major PlcR-dependent haemolysins, sphingomyelinase and anthrolysin $\mathrm{O}$ is enhanced in response to PlcR activation in $B$. anthracis, only anthrolysin $\mathrm{O}$ contributes significantly to lysis of human erythrocytes. In contrast, the toxicity of bacterial culture supernatants from a PlcR-positive strain towards murine macrophages occurred independently of anthrolysin $\mathrm{O}$ expression in vitro and in vivo.
\end{abstract}

\section{INTRODUCTION}

Bacillus cereus and Bacillus anthracis, members of the B. cereus group of bacteria, have high genetic similarity (Ivanova et al., 2003; Read et al., 2003), and may even constitute a single species (Helgason et al., 2000). While $B$. cereus is an opportunistic human pathogen (Drobniewski, 1993), B. anthracis is the aetiological agent of anthrax, a disease with a high lethality in many animal species, including humans (Mock \& Fouet, 2001). The strikingly different behaviour of these closely related species is attributed to $B$. anthracis having several additional genetic features, such as the virulence plasmids pXO1 and pXO2. These plasmids encode genes for the anthrax tripartite toxin, composed of protective antigen (PA), lethal factor (LF) and oedema factor (EF), and genes for the biosynthetic enzymes for the antiphagocytic poly- $\gamma$-D-glutamic acid capsule (Mock \& Fouet, 2001). Another unique genetic trait involves the pleiotropic transcriptional regulator PlcR

‡Deceased

Abbreviations: BMDMs, bone marrow-derived macrophages; LF, lethal factor; LT, lethal toxin; PA, protective antigen.
(Agaisse et al., 1999; Lereclus et al., 1996). In B. cereus, PlcR acts by binding to a well-defined site (the PlcR box), consisting of a $16 \mathrm{bp}$ consensus sequence present in promoter regions of PlcR-regulated genes (Agaisse et al., 1999; Okstad et al., 1999). Furthermore, PlcR action requires the participation of a secreted, processed and reimported heptapeptide derived from PapR (Bouillaut et al., 2008; Lereclus et al., 1996; Okstad et al., 1999; Slamti et al., 2004). Comparative protein and RNA expression profiles of PlcR-positive and -negative B. cereus and Bacillus thuringiensis strains have identified genes regulated by $\mathrm{PlcR}$ that encode collagenases, haemolysins, phospholipases and enterotoxins (Gohar et al., 2002, 2008; Slamti et al., 2004). Conversely, in B. anthracis, a nonsense mutation in plcR results in a premature translational stop (Agaisse et al., 1999; Slamti et al., 2004), and consequently gene expression in B. anthracis differs drastically from that in B. cereus. Certain phenotypic properties of $B$. anthracis, such as lack of haemolytic activity towards erythrocytes (Burdon, 1956), can be attributed to the non-functional PlcR, even though the genes for at least two haemolysins are present in the $B$. anthracis genome. The best-characterized haemolysin of $B$. anthracis is anthrolysin $\mathrm{O}$, a member of the 
cholesterol-dependent family of haemolysins, which causes lysis of a variety of cells (Mosser \& Rest, 2006; Shannon et al., 2003; Tweten, 2005). Although B. anthracis strains are non-haemolytic on blood agar plates, this PlcR-dependent haemolysin is expressed under certain culture conditions as well as in vivo (Ross \& Koehler, 2006; Shannon et al., 2003).

In $B$. anthracis, studies of the putative PlcR regulon have been restricted to sequence searches for PlcR boxes and comparisons with $B$. cereus, and have resulted in the identification of about $50 \mathrm{~B}$. anthracis genes that are potentially subject to regulation by PlcR (Mignot et al., 2001; Rasko et al., 2005; Read et al., 2003). Several studies report the activation of the PlcR regulon in $B$. anthracis (Mignot et al., 2001; Pomerantsev et al., 2003, 2004). One study shows that expression of PlcR in B. anthracis affects sporulation of bacteria containing the virulence plasmid pXO1, but not of bacteria lacking either $\mathrm{pXO1}$ or the $\mathrm{pXO1-}$ encoded regulator AtxA alone, and it has been hypothesized that in $B$. anthracis, functional PlcR is incompatible with AtxA-controlled gene regulation (Mignot et al., 2001). However, subsequent reports have described haemolytic $B$. cereus strains having pXO1-like plasmids (Hoffmaster et al., 2004, 2006; Klee et al., 2006), and a recent report describes the presence of two AtxA homologues in a B. cereus strain containing an active PlcR regulator (Passalacqua et al., 2009). These observations motivated us to readdress the hypothesis that AtxA cannot coexist with an active PlcR system. We have previously shown that the haemolytic activity of $B$. anthracis can be drastically increased upon introduction of plasmid pFP12 containing a PlcR-PapR fusion protein, leading to growth phase-independent activation of the PlcR regulon in $B$. anthracis. This fusion protein contains a full-length PlcR fused to PapR after deletion of the stop codon and one of three repetitive MKK motifs (Pomerantsev et al., 2004). In this study, using plasmid pFP12, we investigated the effect of a functional $\mathrm{PlcR}$ regulon in $B$. anthracis with regard to sporulation, protein expression, anthrolysin-dependent haemolysis, and toxicity towards macrophages in vitro and in vivo.

\section{METHODS}

Bacterial strains, media and growth conditions. Table 1 lists all strains and plasmids used in this study. Bacteria were routinely grown in Luria-Bertani (LB), Brain-Heart Infusion (BHI; BD Biosciences) or nutrient broth with yeast extract (NBY) containing $0.8 \%$ nutrient broth (Sigma), $0.3 \%$ yeast extract, $9 \%$ heat-inactivated equine serum (Hyclone), and $0.6 \% \mathrm{NaHCO}_{3}$ at $37{ }^{\circ} \mathrm{C}$ and 225 r.p.m. in either air or air supplemented with $\mathrm{CO}_{2}$ regulated at $5 \%$ for media supplemented with $\mathrm{NaHCO}_{3}$. AtxA functionality was tested for bacteria grown in NBY broth. Spores were prepared as described previously (Sastalla et al., 2009). For haemolytic studies, bacteria were grown on tryptic soy agar with $5 \%$ sheep blood (both BD Biosciences) or BHI broth. When required, the following antibiotics were added to Escherichia coli or B. anthracis cultures: ampicillin $\left(100 \mu \mathrm{g} \mathrm{ml}^{-1}\right)$, erythromycin $\left(10 \mu \mathrm{g} \mathrm{ml}^{-1}\right)$, kanamycin $\left(20 \mu \mathrm{g} \mathrm{ml}^{-1}\right)$, tetracycline $\left(10 \mu \mathrm{g} \mathrm{ml}^{-1}\right)$ and spectinomycin $\left(150 \mu \mathrm{g} \mathrm{ml}^{-1}\right)$. All antibiotics were purchased from Sigma.
DNA techniques and mutagenesis. For generating an anthrolysin knockout strain, two alo-adjacent fragments were amplified using primers ALL and ALR (fragment aloLF), and primer ARL in combination with ARR (fragment aloRF), harbouring EcoRI and SpeI sites, respectively (Table 1). Amplified fragments were separately cloned into vector pSC, a plasmid allowing sequential generation of markerless deletions using the Cre/Lox system (Pomerantsev et al., 2009). Briefly, plasmid pSC-aloLF was introduced into B. anthracis; electrocompetent cells were prepared as described previously (Park \& Leppla, 2000). Transformants were selected at $30{ }^{\circ} \mathrm{C}$ on erythromycin and chromosomal integration of the plasmid was achieved by a temperature shift to $37^{\circ} \mathrm{C}$. Plasmid pCrePAS was introduced, resulting in excision of the inserted plasmid backbone by Crerecombinase activity, leaving a single loxP site. Plasmid pSC-aloRF was introduced and the $30{ }^{\circ} \mathrm{C} / 37{ }^{\circ} \mathrm{C}$ selection cycle followed by introduction of pCrePAS was repeated, resulting in excision of residual vector and the entire alo gene from the bacterial chromosome.

Plasmid pFP12 (Pomerantsev et al., 2004) was introduced by electroporation. To verify the presence (i.e. retention) of pXO1 in B. anthracis, bacteria were resuspended in Tris/EDTA buffer (Quality Biological), boiled for $2 \mathrm{~min}$, and supernatants were used as template for the PCR using Taq polymerase (Qiagen) and PA (pagA) gene primers PA1 and PA2 (Table 1). For sequencing of atxA, primers AtxSeq1 and AtxSeq5 generated a $1742 \mathrm{bp}$ PCR product that was subsequently used as template for sequencing reactions using primers AtxSeq1, AtxSeq2, AtxSeq3, AtxSeq4 and AtxSeq5.

Sporulation efficacy. Bacteria were grown on sporulation agar (Sastalla et al., 2009) for 5 days, analysed microscopically using a Nikon Eclipse TE200U microscope, scraped off the plates, and resuspended in $1 \mathrm{ml}$ sterile distilled water. Spore suspensions were heat-treated at $65{ }^{\circ} \mathrm{C}$ for $30 \mathrm{~min}$ and plated on LB agar. The percentage of viable spores was calculated using the following equation:

(c.f.u. after heat treatment/c.f.u. before heat treatment) $\times 100$

2D SDS-PAGE and protein identification. Bacteria were grown to the late exponential/early stationary growth phase and centrifuged at $9000 \mathrm{~g}$, and filter-sterilized supernatants were precipitated with TCA (10\% final concentration), washed in ice-cold acetone and resuspended in 2D-rehydration buffer [8 M urea, $2 \%$ (w/v) CHAPS, $0.5 \%$ (v/v) ZOOM carrier ampholytes for $\mathrm{pH}$ range 3-10 (Invitrogen), $0.002 \%$ bromophenol blue, $20 \mathrm{mM}$ DTT]. Proteins were quantified using 2D Quant (Amersham), and $50 \mu \mathrm{g}$ was separated on a NuPAGE gel using the IPG Runner system (Invitrogen) according to the manufacturer's recommendations. Gels were Coomassie-stained and spots of interest were excised, trypsin-digested and analysed by MS. Retrieved data were searched against the $B$. anthracis Ames strain database using Mascot as the search engine. Peptides with a Mascot protein score higher than 60 were considered significant, and the most abundant protein present in each spot is listed in Table 2. MSMS analysis was performed at the National Institute of Allergy and Infectious Diseases (NIAID) Research Technology Branch (RTB) Core Facility. Protein alignments and sequence analyses were performed using Lasergene MEGALIGN, PSORTb (Gardy et al., 2005), SignalP (Emanuelsson et al., 2007), Prosite (http://expasy.org/prosite) and TMpred (http://www.ch.embnet.org).

Western blotting. Filter-sterilized supernatants of bacterial cultures grown in NBY medium to stationary phase were separated on NuPAGE gels (Invitrogen) and blotted onto nylon membranes (Osmonics). Membranes were incubated with $2 \mu \mathrm{g}$ mouse monoclonal antibodies $\mathrm{ml}^{-1}$ against either PA (PA-05-A-G1) or LF (LF-03-A-G1) (both from the Naval Medical Research Center) in $1 \%$ skim milk, and anti-mouse IgG conjugated to horseradish peroxidase (KPL) was used as secondary antibody. Membranes were developed using a tetramethylbenzidine (TMB) peroxidase substrate (KPL). 
Table 1. Bacillus strains, plasmids and primers used in this study

Restriction sites are underlined.

\begin{tabular}{|c|c|c|}
\hline Strain, plasmid or primer & Relevant characteristic(s) & Source or reference \\
\hline \multicolumn{3}{|l|}{ B. anthracis strains } \\
\hline Sterne $34 \mathrm{~F} 2$ & $\mathrm{pXO}^{+}, \mathrm{PlcR}^{-}$ & \\
\hline SdT & $34 \mathrm{~F} 2, \mathrm{pXO1}^{-}, \mathrm{PlcR}^{-}$ & Ivins et al. (1986) \\
\hline SdT12 & $34 \mathrm{~F} 2, \mathrm{pXO}^{-}$, harbouring $\mathrm{pFP} 12, \mathrm{PlcR}^{+}$ & Pomerantsev et al. (2004) \\
\hline Sterne pFP12 & 34F2 harbouring pFP12, $\mathrm{PlcR}^{+}\left(\mathrm{pXO1}{ }^{+}\right)$ & This study \\
\hline Sterne $\Delta \mathrm{ALO}$ & $34 \mathrm{~F} 2$, anthrolysin-negative $\left(\mathrm{pXO1}^{+}\right)$ & This study \\
\hline Sterne $\triangle \mathrm{ALO}$ pFP12 & $\begin{array}{l}\text { 34F2, anthrolysin-negative, harbouring pFP12, } \\
\mathrm{PlcR}^{+}\left(\mathrm{pXO}^{+}\right)\end{array}$ & This study \\
\hline \multicolumn{3}{|l|}{ B. cereus strain } \\
\hline 569 & & Reddy et al. (1987) \\
\hline \multicolumn{3}{|l|}{ E. coli strains } \\
\hline TOP10 & & Invitrogen \\
\hline XL2-Blue & & Stratagene \\
\hline SCS110 & & Stratagene \\
\hline \multicolumn{3}{|l|}{ Plasmids } \\
\hline pFP12 & Encodes a PlcR-PapR fusion protein & Pomerantsev et al. (2004) \\
\hline pSC & Contains loxP site for $B$. anthracis mutagenesis & Pomerantsev et al. (2009) \\
\hline pSC-aloLF & pSC with ALL and ALR-derived amplicon & This study \\
\hline pSC-aloRF & pSC with ARL and ARR-derived amplicon & This study \\
\hline pCrePAS & Contains Cre recombinase & Pomerantsev et al. (2009) \\
\hline Primers & Sequence $\left(5^{\prime}-3^{\prime}\right)$ & Relevant property \\
\hline ALL & ccccgaattcagaagcagaaagcccegcat & Amplification of aloLF \\
\hline ALR & ccccactagtcggcttgtgtttctgcaaaa & \\
\hline ARL & ccccgaattcttgcatgggaatggtggaga & Amplification of aloRF \\
\hline ARR & ccccactagttgcccccaaatagacttcaa & \\
\hline PA1 & gattcaggcagaagttaaacagg & Amplification of pagA \\
\hline PA2 & cccaccaatatcaaagaacg & \\
\hline AtxSeq1 & tgctatttcaatagaagaaacaaaaa & Used to sequence AtxA \\
\hline AtxSeq2 & tccaagatggaggaatttcg & \\
\hline AtxSeq3 & tgcaatccttagctcaaaactc & \\
\hline AtxSeq 4 & cetttggctaaagaaattacc & \\
\hline AtxSeq5 & tatccatcgctatgcaggtg & \\
\hline BA1995fw & cgcgtcatctgtatcttatgc & Used for BA1995 RT-PCR \\
\hline BA1995rv & agcttgtccatttcctgtgc & \\
\hline Sph1 & tatccgaattggggacaaag & Used for sph RT-PCR \\
\hline Sph2 & gaagcgtgcaatgttttgaa & \\
\hline Alo1 & tgtggctggagcagtagatg & Used for alo RT-PCR \\
\hline Alo2 & cactcgtttcgacgctgtta & \\
\hline GyrAfw & aaaacctgtgcatcgtaggg & Used for gyrA RT-PCR \\
\hline GyrArv & acattagcattggcatcacg & \\
\hline
\end{tabular}

RNA isolation and RT-PCR. Cultures grown in BHI to late exponential/stationary phase were centrifuged, and RNA was stabilized using RNA Protect Bacteria reagent (Qiagen) before further isolation using a Qiagen RNeasy kit. Further preparation of RNA and RT-PCR was performed as described previously (Sastalla et al., 2009). To amplify targeted mRNAs, the following oligonucleotides were used: primers BA1995fw in combination with BA1995rv, primers Sph1 and Sph2, and primers Alo1 and Alo2 (Table 1). Internal control reactions were performed with primers GyrAfw and GyrArv as described previously (Sastalla et al., 2009). Band intensities were quantified using ImageJ version $1.40 \mathrm{~g}$.

Haemolytic assays. Human blood obtained from the National Institutes of Health (NIH) Blood Bank was washed with PBS and resuspended in PBS to the original volume. A $40 \mu \mathrm{l}$ volume of blood was mixed with $160 \mu \mathrm{l}$ culture supernatant obtained from bacteria grown in BHI medium to which $20 \mathrm{mM}$ cysteine had been added, incubated at $37{ }^{\circ} \mathrm{C}$ for $45 \mathrm{~min}$ and centrifuged at $500 \mathrm{~g}$, and the $A_{540}$ of supernatants was measured in a SpectraMAX 190 plate reader (Molecular Devices). A haemolysis value of $100 \%$ corresponded to haemolysis obtained by addition of $0.1 \%$ SDS in BHI broth. For some experiments, cholesterol at a final concentration of $25 \mu \mathrm{M}$ was added.

Preparation and use of bone marrow-derived macrophages (BMDMs). Bone marrow cells from 8-14-week old DBA/2J mice (Jackson Laboratories) were cultured in two parts complete Dulbecco's modified Eagle's medium (DMEM) [10\% fetal bovine serum (FBS), $10 \mathrm{mM}$ HEPES, $50 \mu \mathrm{g}$ gentamicin $\mathrm{ml}^{-1}$ ] and one part L929 cell culture 
Table 2. Proteins identified by $2 \mathrm{D}$ gel electrophoresis

\begin{tabular}{|c|c|c|c|c|}
\hline Gene ID & Description & Loc.* & $M_{\mathbf{r}}$ & pI \\
\hline \multicolumn{5}{|c|}{ B. anthracis } \\
\hline BA0885 & S-layer protein SAP & $\mathrm{CW}$ & 86.6 & 7.6 \\
\hline BA0887 & S-layer protein EA1 & CW & 91.4 & 5.8 \\
\hline BA0796 & Conserved hypothetical protein & $\mathrm{E}$ & 40.8 & 9.6 \\
\hline BA1449 & Peptidase, M23/M37 family & E & 46.2 & 6.7 \\
\hline BA1952 & NLP/P60 family protein & CW & 43.9 & 10.2 \\
\hline \multicolumn{5}{|c|}{$\begin{array}{l}\text { B. anthracis } \\
\text { pFP12 }\end{array}$} \\
\hline BA0267 & GroEL, $60 \mathrm{kDa}$ chaperonin $\dagger$ & $\mathrm{C}$ & 57.4 & 4.5 \\
\hline BA0555 & Putative collagenase $\dagger$ & $\mathrm{E}$ & 109.9 & 5.0 \\
\hline BA0620 & Putative 8-amino-7-oxononanoate synthase & $\mathrm{C}$ & 43.1 & 5.7 \\
\hline BA0670 & Putative transaldolase & $\mathrm{C}$ & 23.1 & 5.3 \\
\hline BA0672 & InhA metalloprotease $\dagger$ & E & 87.9 & 5.7 \\
\hline BA0677 & Phospholipase C†末 & $\mathrm{E}$ & 32.4 & 7.9 \\
\hline BA1887 & Enterotoxin $\dagger \ddagger$ & E & 43.6 & 4.8 \\
\hline BA1995 & Putative protease $\dagger \neq$ & $\mathrm{E}$ & 43.9 & 9.0 \\
\hline BA2032 & Hypothetical protein $\neq$ & E & 16.2 & 8.9 \\
\hline BA3162 & Putative 5 '-nucleotidase $\dagger$ & $\mathrm{C}$ & 57.8 & 6.5 \\
\hline BA3891 & Phosphatidylinositol diacylglycerol-lyase $\dagger \ddagger$ & E & 38.1 & 7.9 \\
\hline BA3962 & Ribosome recycling factor & $\mathrm{C}$ & 20.7 & 5.5 \\
\hline BA3964 & Translation elongation factor Ts & $\mathrm{C}$ & 32.4 & 5.0 \\
\hline BA4181 & $\begin{array}{l}\text { Pyruvate dehydrogenase complex E3 component, } \\
\text { dihydrolipoamide dehydrogenase } \dagger\end{array}$ & $\mathrm{C}$ & 49.5 & 5.0 \\
\hline BA4184 & Pyruvate dehydrogenase complex E1 component, alpha subunit & $\mathrm{C}$ & 41.4 & 5.6 \\
\hline BA4194 & $\begin{array}{l}\text { Putative 2,3,4,5-tetrahydropyridine-2-carboxylate } N \text { - } \\
\text { succinyltransferase }\end{array}$ & $\mathrm{C}$ & 25.7 & 5.2 \\
\hline BA4499 & Superoxide dismutase, $\mathrm{Mn} \dagger$ & $\mathrm{E}$ & 22.7 & 5.3 \\
\hline BA5130 & Phosphoglucose isomerase & $\mathrm{C}$ & 50.3 & 4.8 \\
\hline BA5364 & Enolase $\dagger$ & $\mathrm{C}$ & 46.4 & 4.4 \\
\hline BA5470 & Sulfatase & MA & 73.5 & 6.4 \\
\hline BA5580 & Fructose-bisphosphate aldolase, class II & $\mathrm{C}$ & 30.7 & 4.8 \\
\hline
\end{tabular}

${ }^{*}$ Cellular location: C, cytoplasmic/unknown; CW, cell wall; MA, membrane-associated; E, extracellular.

$\dagger$ Protein found in supernatants of B. cereus.

$\$$ PlcR box present.

supernatant for 7 days. Cells were washed and resuspended in serumfree DMEM, and $80 \mu \mathrm{l}$ aliquots were transferred to 96-well microtitre plates. Filter-sterilized bacterial culture supernatants $(25 \mu \mathrm{l})$ harvested from BHI-grown bacteria were added, and plates were incubated for $4 \mathrm{~h}$ at $37{ }^{\circ} \mathrm{C}$ and $5 \% \mathrm{CO}_{2}$. For some experiments, supernatants were heat-treated at $95{ }^{\circ} \mathrm{C}$ for $10 \mathrm{~min}$ before addition to cells. To determine the viability of cells, 3-(4,5-dimethylthiazol-2-yl)-2,5-diphenyltetrazolium bromide (MTT; Sigma) was added at a final concentration of $1 \mathrm{mg} \mathrm{ml}^{-1}$, and pictures of the stained (viable) cells were taken after incubation for $45 \mathrm{~min}$ at $37{ }^{\circ} \mathrm{C}$ in $5 \% \mathrm{CO}_{2}$. The amount of formazan produced by viable cells was quantified at $590 \mathrm{~nm}$ after removal of medium and addition of $50 \mu \mathrm{l}$ dissolving buffer containing $0.5 \%$ SDS and $25 \mathrm{mM} \mathrm{HCl}$ dissolved in $90 \%$ 2-propanol.

Intraperitoneal treatment of mice and isolation of cells. DBA/2J mice (8-14 weeks old, $n=2$ in at least three independent studies) received intraperitoneal injections of $500 \mu$ filter-sterilized bacterial culture supernatants or BHI alone. After an incubation period of $45 \mathrm{~min}$, intraperitoneal lavages were performed with PBS containing $1 \%$ BSA (PBSA). For some experiments, digital images of lavage samples were taken, or samples were centrifuged, treated twice with ammonium chloride lysing buffer (ACK; Invitrogen) to eliminate red blood cells, washed two more times with PBSA, and resuspended in $600 \mu \mathrm{l}$ of the same buffer. Animal experiments described in this and the previous section were performed according to NIH and Animal Welfare Act guidelines and approved by the Animal Care and Use Committee of the National Institute of Allergy and Infectious Diseases, National Institutes of Health.

Flow cytometry analyses. Cell suspensions in PBSA were incubated with rat anti-mouse CD32 antibodies (BD Biosciences) for blocking of the Fc receptor, followed by allophycocyanin (APC)-conjugated anti-mouse F4/80 (AbD Serotec) to label macrophages. After incubation for $30 \mathrm{~min}$ at $4{ }^{\circ} \mathrm{C}$, cells were washed with PBSA and subjected to flow cytometric analysis using an LSR II flow cytometer (BD Biosciences). Data were analysed either in DIVA software (BD Biosciences) or in FlowJo version 7 (Tree Star).

Statistics. A $t$ test or one-way ANOVA was performed for unpaired data (sporulation efficacy and macrophage retrieval from mice), and a paired $t$ test was performed for paired data (cholesterol inhibition and in vitro treatment of macrophages with heat-inactivated/non-heated supernatant) in GraphPad Prism version 5.01. 


\section{RESULTS}

\section{AtxA regulation does not interfere with sporulation in B. anthracis expressing PIcR-PapR}

We readdressed the hypothesis that expression of functional $\mathrm{PlcR}$ is incompatible with pXO1-encoded, AtxAcontrolled gene regulation. Expression of the PlcR-PapR fusion protein encoded by plasmid pFP12 (Pomerantsev et al., 2004) in B. anthracis strain Sterne 34F2 resulted in colonies that were highly haemolytic on blood agar plates (Fig. 1a). Microscopic analyses of the parental and PlcR-PapR-positive (Sterne pFP12) strains surprisingly showed that both strains readily formed spores (Fig. 1b).

(a)

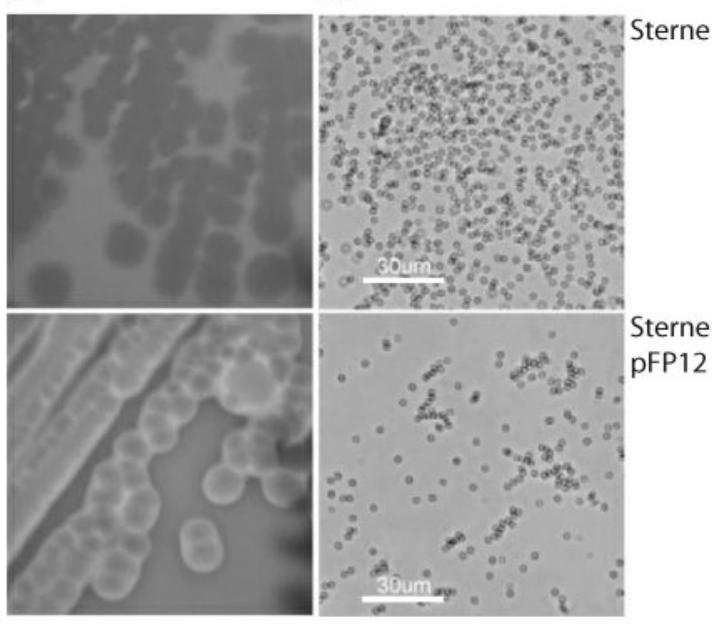

(c)

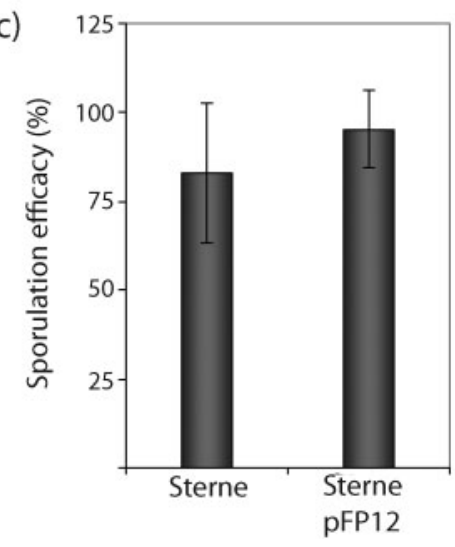

(d)

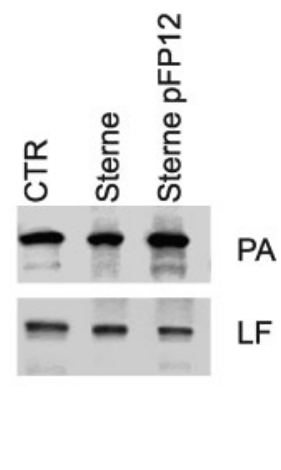

Fig. 1. Characterization of PlcR-PapR-expressing strains. (a) Haemolysis on sheep blood agar by Sterne strain and Sterne expressing PlcR-PapR. (b) Microscopic analysis of spores formed by Sterne and the isogenic PlcR-PapR-expressing strain. (c) Efficacy of sporulation. Shown are mean $\pm S D$ from one representative experiment out of three. (d) Western blot analysis of AtxA activity by determining $\mathrm{PA}$ and LF expression in response to bicarbonate/ $\mathrm{CO}_{2}$. Control (CTR) bands represent $1 \mu \mathrm{g}$ recombinant protein.
Quantification of sporulation efficacy verified that both strains sporulated with similar high efficacies (Fig. 1c).

Growth of B. anthracis at elevated temperatures (above $43{ }^{\circ} \mathrm{C}$ ) can induce loss of the virulence plasmid pXO1 (Mikesell et al., 1983). To exclude the possibility that pXO1 was lost during growth, we verified the presence of pXO1 by PCR using oligonucleotides specific for the PA gene (pagA) (data not shown).

To determine whether a point mutation in the atxA gene could have caused inactivation of the regulator, we sequenced the entire atxA gene in Sterne 34F2 and Sterne pFP12. The sequences were $100 \%$ identical to the annotated Sterne atxA sequence (data not shown), including $470 \mathrm{bp}$ of the upstream promoter region, thereby excluding the possibility that AtxA expression in the PlcR-PapR-positive strain was silenced by mutation.

To rule out the possibility that other unrecognized changes may have inactivated AtxA regulation, we investigated the functionality of AtxA in both the wild-type and pFP12containing strains. It has been established that expression of $B$. anthracis toxin genes is enhanced by elevated bicarbonate $/ \mathrm{CO}_{2}$ (Leppla, 1988). This higher expression requires AtxA, even though transcription of the regulator itself is not affected by bicarbonate/ $\mathrm{CO}_{2}$ levels (Dai \& Koehler, 1997). Analysis of PA and LF expression in Sterne and Sterne pFP12 grown in the presence of bicarbonate/ $\mathrm{CO}_{2}$ showed that both toxins are expressed at similar levels in both strains, verifying that AtxA is functional in both strains (Fig. 1d). These results show that strain Sterne 34F2 is able to sporulate when PlcR-PapR and AtxA are simultaneously active, and that there is no incompatibility of these two regulators, at least in the strains we investigated here.

\section{Activation of the PIcR regulon in B. anthracis leads to expression of proteins found in the secretome of $B$. cereus}

While the secretomes of $B$. cereus and $B$. thuringiensis are highly similar, $B$. anthracis shows a distinctively different secretome, probably due to the presence of an inactive PlcR (Chitlaru et al., 2006; Gohar et al., 2002). Thus, we assessed the influence of heterologous PlcR-PapR activation in $B$. anthracis on the composition of the bacterial secretome by 2D SDS-PAGE. Fig. 2 shows that the PlcR-expressing $B$. anthracis derivative has a secreted protein profile distinctively different from that of wild-type $B$. anthracis. We selected proteins that appeared unique for MS analysis (Fig. 2, Table 2). We found S-layer proteins SAP/EA1 to be highly abundant in B. anthracis lacking PlcR-PapR, indicating either lower expression or degradation by PlcRregulated proteases. We identified several proteins found in supernatants of $B$. cereus, including homologues of an enterotoxin component (BA1887), phospholipases (BA3891 and BA0677), InhA metalloprotease (BA0672) and a putative protease (BA1995). A protein of unknown function 


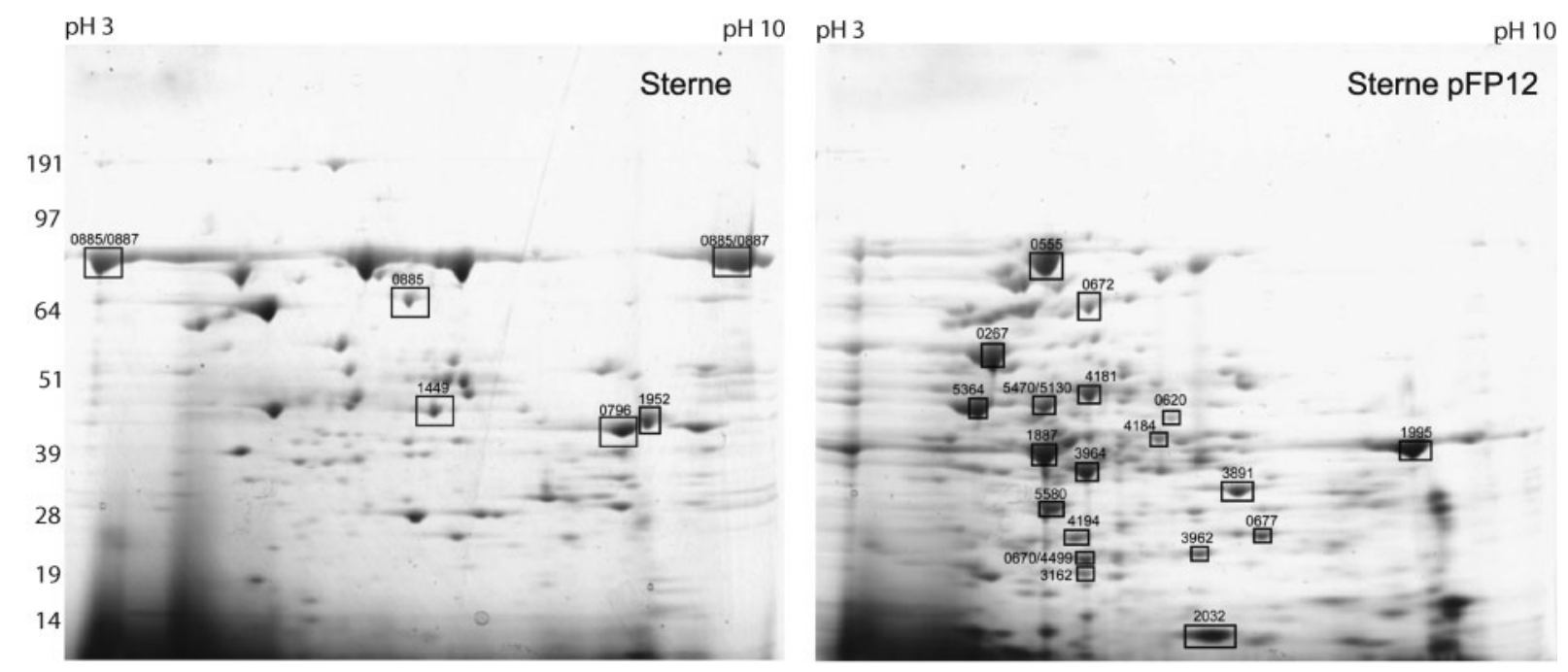

Fig. 2. 2D SDS-PAGE separation of proteins secreted by Sterne and Sterne pFP12 grown in BHI broth to late exponential phase. Boxed spots indicate proteins identified by MS. Proteins are numbered according to $B$. anthracis Ames (BA) gene ID. See Table 2 for gene descriptions. Molecular mass (in $\mathrm{kDa}$ ) is given on the left and the $\mathrm{pH}$ range is indicated at the top.

(BA2032) showed high expression in PlcR-activated $B$. anthracis, and interestingly the gene is present in all $B$. anthracis strains sequenced to date, but in only four of the sequenced B. cereus strains (W-8, AH820, E33L and 95/8201). Analysis of the upstream regions of all identified proteins for a putative PlcR binding site showed that five of them indeed harboured a PlcR box (Table 2). These results demonstrate the effect that activation of the $\mathrm{PlcR}$ regulon has on the expression profile of $B$. anthracis, and show that expression of a PlcR-PapR fusion protein causes the secretome of $B$. anthracis to acquire similarities to that of $B$. cereus.

\section{Expression of the putative protease BA1995 is enhanced by PIcR-PapR}

One difference between the two $B$. anthracis secretomes examined in Fig. 2 was a protein band of $40-50 \mathrm{kDa}$ that was highly abundant only in the PlcR-PapR-positive strain (Fig. 2). MS identified this as the putative protease BA1995, and matched peptides from the analysis are shown in Fig. 3(a). This protein belongs to the transglutaminase-like superfamily (PFAM 01841) and a homologue is present in B. cereus (BC1991). PlcR box homology searches had failed to identify BA1995 and BC1991 as likely to be regulated by PlcR, but our data suggested that BA1995 production might be either directly or indirectly regulated by PlcR. Quantification of the $B A 1995$ gene transcript in these two strains relative to the housekeeping gene gyrA showed the amount of transcript for BA1995 to be about eightfold higher in the pFP12containing strain than in the wild-type, indicating that $\mathrm{PlcR}$ can regulate this gene (Fig. 3b). Interestingly, even though the wild-type strain does not have a functional PlcR, low levels of BA1995 mRNA were observed, indicating weak transcription independent of PlcR.
Examination of the region upstream of the BA1995 gene identified a near-perfect PlcR box 82 bases upstream of the start codon (Fig. 3c). The sequence differed from the consensus sequence by a residue that is generally conserved. The region upstream of the B. cereus BC1991 gene showed the same deviation from the consensus, which might explain why previous sequence searches failed to identify this gene as being PlcR-dependent.

\section{In B. anthracis, transcription of the cytolysins sphingomyelinase and anthrolysin is induced by PIcR-PapR}

The genome of $B$. anthracis encodes fewer active haemolysins than that of $B$. cereus. The genes for sphingomyelinase (sph) and anthrolysin (alo) are intact, whereas the homologues of B. cereus haemolysins II ( $h l y \mathrm{II})$ and III ( $h l y \mathrm{III}$ ) are truncated (Klichko et al., 2003). Phospholipase C (PC-PLC; BA0677), which we were able to detect in culture supernatants of $B$. anthracis having an activated PlcR regulon (Fig. 2), is non-haemolytic on its own (Beecher \& Wong, 2000). In B. cereus, the sph and clo genes are regulated by PlcR (Gohar et al., 2008; Lereclus et al., 1996; Pomerantsev et al., 2004; Slamti et al., 2004), and we confirmed that expression of PlcR-PapR in $B$. anthracis results in a haemolytic phenotype. To determine the contribution of each haemolysin, we measured mRNA levels at different time points during growth. In the absence of PlcR, only weak expression of sphingomyelinase and anthrolysin was observed (Fig. 4a). However, constitutive expression of the PlcR-PapR fusion protein greatly enhanced transcription of both haemolysins in the wild-type Sterne strain (Fig. 4a). These results show that both haemolysins are transcribed in the 
(a)

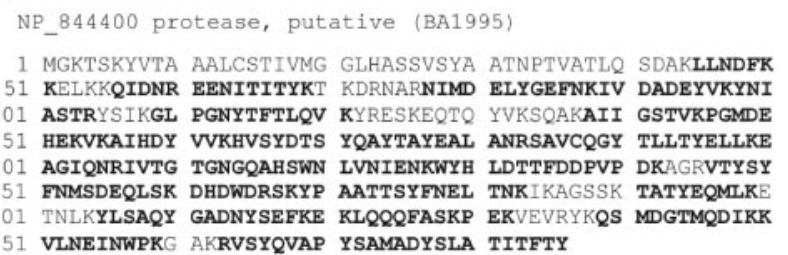

(b)

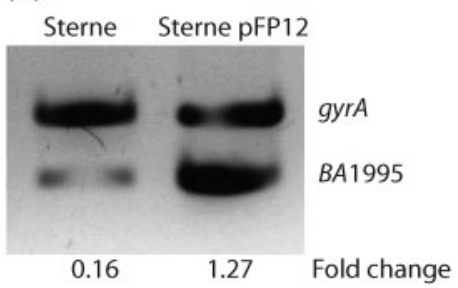

(c)

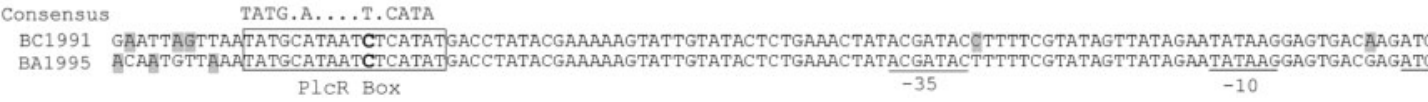

Fig. 3. Identification and analysis of the PlcR-dependent protease BA1995. (a) Putative protease BA1995 identified by 2D SDSPAGE in supernatants of Sterne pFP12. Matched peptides are shown in bold type. (b) Semiquantitative RT-PCR of BA1995 transcription in response to PlcR-PapR expression (lower band). The gene for gyrase A (gyrA) served as internal control. Fold change indicates gyrA-normalized differences in BA1995 transcript between Sterne and Sterne pFP12. (c) Sequence comparisons of the PlcR box present in the promoter region of $B A 1995$ of $B$. anthracis strain Ames and the homologue BC1991 in B. cereus. The PlcR consensus sequence is indicated. The nucleotide differing from the consensus is shown in bold type, nucleotides differing between $B$. cereus and $B$. anthracis are shaded, and putative $-10,-35$ regions and the start codon (ATG) are indicated.

PlcR-PapR-positive B. anthracis, and only weakly in the wild-type strain.

\section{PIcR-PapR-expressing $B$, anthracis lyses human red blood cells, with anthrolysin being the main contributor}

Although B. anthracis is considered non-haemolytic (Burdon, 1956), it has been reported that growth in rich medium causes anthrolysin production that leads to haemolysis (Shannon et al., 2003). To further evaluate the contribution of anthrolysin to haemolysis, we generated an anthrolysin gene knockout mutant of the Sterne strain and investigated the haemolytic behaviour towards human erythrocytes before and after introduction of pFP12. Supernatants of the pFP12-containing strain were highly haemolytic throughout the growth cycle (Fig. 4b). Supernatants of the PlcR-PapR-negative wild-type strain (a)

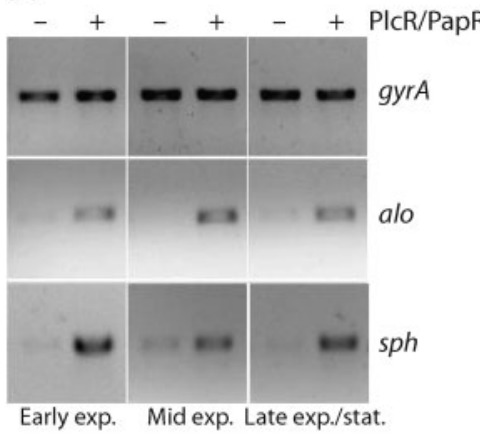

(b)

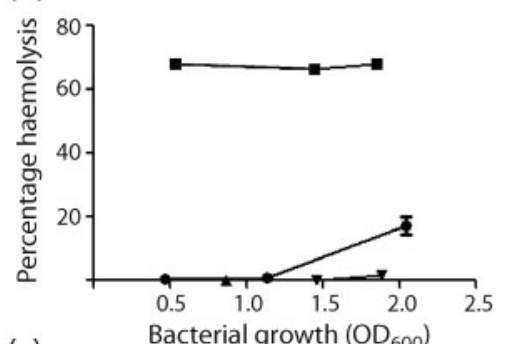

(c)

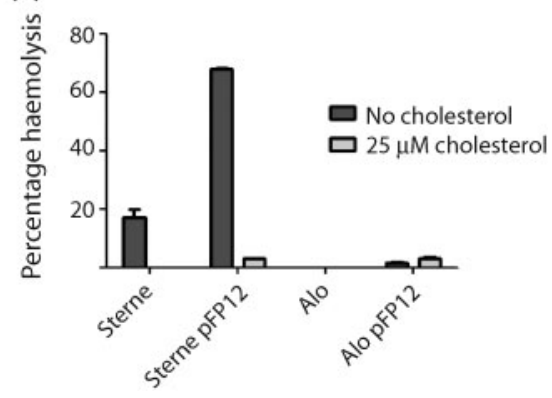

Fig. 4. Transcription and expression of haemolysins in PlcR-PapR-expressing $B$. anthracis. (a) Semiquantitative RT-PCR of $B$. anthracis putative haemolysins anthrolysin (a/o) and sphingomyelinase $(s p h)$ in response to PlcR-PapR expression in Sterne and Sterne pFP12. Transcript levels of bacteria harvested at different growth phases were compared with those for gyrase A (gyrA). exp., exponential; stat., stationary. (b) Haemolytic activity (as a percentage) towards human erythrocytes of bacterial supernatants derived from Sterne or anthrolysin knockout mutant (Alo) in the presence or absence of PlcRPapR. Supernatants were harvested at different $\mathrm{OD}_{600}$. Shown are mean $\pm \mathrm{SD}$ from one representative experiment out of three. $\bullet$, Sterne; $\boldsymbol{\square}$, Sterne pFP12; $\boldsymbol{\Delta}$, Alo; $\boldsymbol{\nabla}$, Alo pFP12. (c) Haemolytic activity (\%) of bacterial supernatants in the presence or absence of $25 \mu \mathrm{M}$ cholesterol. Shown are mean \pm SD from one representative experiment out of three. 
showed a much lower degree of haemolysis that was observed only after the onset of stationary phase. In contrast, the anthrolysin knockout strain did not show haemolysis at any point in the growth cycle, and expression of PlcR-PapR in the anthrolysin knockout led to only a very low level of haemolysis $(1.9 \% \pm 0.05)$. Complementation of the knockout by expressing alo on a plasmid under the control of the PA promoter restored haemolysis to the level seen for the Sterne pFP12 transformant (data not shown).

Anthrolysin is a cholesterol binding haemolysin, and addition of cholesterol inhibits its activity (Tweten, 2005). Cholesterol strongly blocked haemolysis by both wild-type Sterne and the pFP12-containing strain (Fig. 4c). No haemolysis was observed for the anthrolysin knockout, and expression of PlcR-PapR in this mutant could not restore the high haemolytic phenotype observed for Sterne pFP12 in the absence of cholesterol. The small residual haemolytic activities of about $3 \%$ seen for both the PlcR-PapR-positive anthrolysin knockout and the PlcR-PapR-positive anthrolysin-containing strain show that other PlcR-dependent haemolysins contribute only slightly. In summary, these results demonstrate that anthrolysin is the main contributor to haemolysis of human erythrocytes by PlcR-PapR-positive and -negative B. anthracis and that other PlcR-regulated haemolysins, such as sphingomyelinase, have a negligible effect.

\section{Supernatants of PIcR-PapR-positive B. anthracis kill BMDMs in an anthrolysin-independent manner}

In vitro, recombinant anthrolysin lyses a variety of cells, including human leukocytes, monocytes and lymphocytes (Mosser \& Rest, 2006). We investigated whether anthrolysin contributes to macrophage toxicity, and for comparison included culture supernatants derived from a B. cereus strain. To exclude the known cytotoxic effects caused by lethal toxin (LT) on certain macrophages, BMDMs from LT-resistant DBA mice (McAllister et al., 2003) were used. Macrophages treated with $B$. cereus culture supernatants were efficiently lysed, as seen by the lack of purple formazan when compared with cells treated with medium alone (Fig. 5a), and surprisingly, culture supernatants derived from the PlcR-PapR-negative Sterne and anthrolysindeficient strains were non-toxic (Fig. 5a, b). Treatment of $\mathrm{BMDMs}$ with culture supernatants from $\mathrm{pFP} 12$-containing bacteria, however, resulted in death of about $50 \%$ of macrophages (Fig. 5b). Heat treatment of supernatants prior to incubation with BMDMs abolished the cytotoxic effect (Fig. 5b). These data show that PlcR-dependent proteins other than LT and anthrolysin are responsible for the observed killing.

\section{Intraperitoneal toxicity of PIcR-PapR-positive $B$. anthracis is not dependent on anthrolysin}

Since the PlcR-PapR-positive B. anthracis supernatants efficiently killed murine macrophages in an in vitro setting, we hypothesized that similar cytotoxicity might occur in vivo. Mice having LT-resistant macrophages were injected intraperitoneally with cell-free culture supernatants derived from various strains. After $45 \mathrm{~min}$, peritoneal contents were collected by lavage. Interestingly, in mice that received injections of supernatants from PlcR-PapR-positive bacteria, we observed intraperitoneal bleeding, as indicated by the presence of erythrocytes (Fig. 6a). This haemorrhaging was highly reproducible and was never detected following injection of supernatants derived from PlcR-PapR-negative bacteria. We also observed this bleeding when B. cereusderived supernatants were injected (results not shown).

The specific cell populations present in the peritoneal washes were assessed by flow cytometry. Two cell populations were missing in the peritoneal washes derived from mice that received injections of supernatants from PlcR-PapR-positive, but not from PlcR-PapR-negative, strains (Fig. 6b). Closer characterization of cell populations using an antibody that binds to the macrophage-specific F4/80 receptor identified the two missing populations as macrophages (Fig. 6c). Quantification of F4/80-positive cells showed a significant $(P<0.0001$, one-way ANOVA and $P<0.05$ Tukey test) fivefold reduction in the numbers of macrophages retrieved from mice that received pFP12containing B. anthracis culture supernatants (Fig. 6d). In concurrence with in vitro results, this reduction does not appear to depend on anthrolysin, since macrophages were also depleted when the anthrolysin gene was absent (Fig. 6b-d). The toxicity appears to depend on one or more proteins, since heat treatment of supernatants prior to injection completely abolished cytotoxicity (results not shown).

\section{DISCUSSION}

Besides the presence of the two large virulence plasmids, truncation of the major regulator PlcR is one of the major differences setting $B$. anthracis apart from other members of the B. cereus group (Kolstø et al., 2009). The nonsense mutation leading to truncation of the PlcR protein is identical in all B. anthracis isolates so far investigated, thereby strongly suggesting that it is a distinctive and defining trait of this species (Slamti et al., 2004). Inactivation of PlcR has a major effect on protein expression (Gohar et al., 2002) in B. cereus; however, little is known about the $\mathrm{PlcR}$ regulon in $B$. anthracis, including the identity of genes that retain the potential to be activated by $\mathrm{PlcR}$. To elucidate the role of $\mathrm{PlcR}$ in $B$. anthracis, we expressed an active PlcR-PapR hybrid protein from a plasmid (Pomerantsev et al., 2004), thereby activating the latent PlcR regulon. An earlier study suggested that expression of PlcR in B. anthracis leads to interference with the pXO1-dependent AtxA regulon, as indicated by the inability of bacteria harbouring both regulators to sporulate (Mignot et al., 2001). Surprisingly, the B. anthracis strain containing both regulators that we describe here readily formed spores. This finding deserves further study in 
(a)
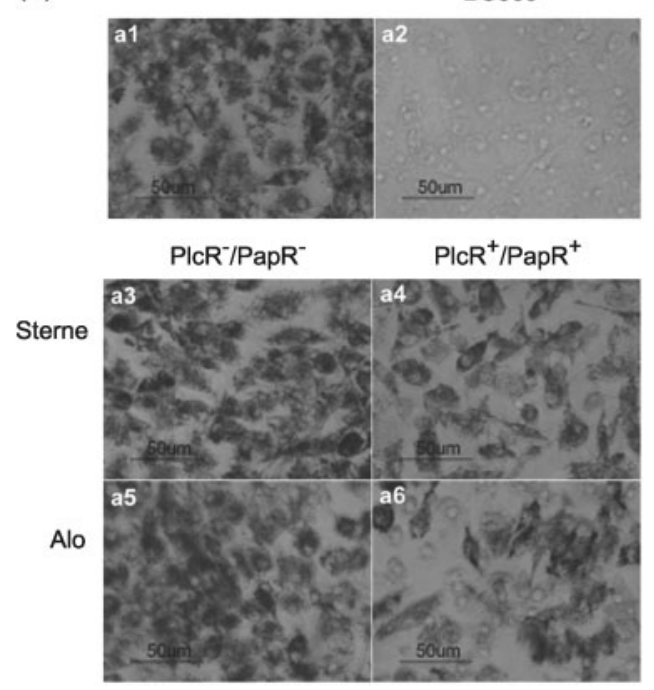

(b)

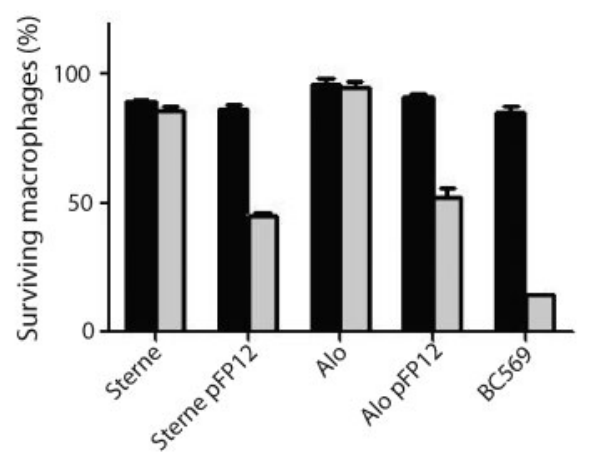

Fig. 5. In vitro toxicity towards macrophages of proteins secreted by Bacillus. (a) MTT stain of BMDMs treated for $4 \mathrm{~h}$ with cellfree bacterial culture supernatants derived from late exponential phase B. cereus BC569 (a2), B. anthracis (a3), B. anthracis pFP12 (a4), B. anthracis anthrolysin knockout (a5), B. anthracis anthrolysin knockout harbouring pFP12 (a6), and broth-treated control (N.T.) (a1). (b) Quantification of surviving macrophages treated with bacterial culture supernatants before (N.T.; grey bars) and after (H.I.; black bars) heat treatment at $95{ }^{\circ} \mathrm{C}$ for $10 \mathrm{~min}$. Shown are mean \pm SD from one representative experiment out of three.

strains having the PlcR-PapR regulon activated in a manner different from that of the fusion protein encoded by pFP12.

We also compared the secretomes of PlcR-PapR-expressing strains with those of their PlcR-PapR-negative counterparts. The secretomes of $B$. anthracis having active or inactive PlcR were distinctively different, and in the former we identified five proteins harbouring a PlcR binding site in their promoter region. It is likely that analysis of all protein spots present in the secretome of PlcR-active B. anthracis would have identified even more such proteins. Furthermore, we detected many proteins in the secretome of PlcR-expressing B. anthracis that are found in culture supernatants of PlcR-active B. cereus (Gohar et al., 2002, 2005). For example, the B. cereus homologue of the putative protease BA1995 was present in supernatants of PlcR-expressing B. anthracis, but absent in supernatants derived from the PlcR-negative parent strain. The database annotations available for BA1995 note similarities to a transglutaminase-like protease (Makarova et al., 1999), although the function of the protein is unknown. Our hypothesis that this protein is dependent on $\mathrm{PlcR}$ was confirmed by semiquantitative RT-PCR. Closer analysis of the BA1995 promoter region revealed a PlcR box having one mismatch at position 11 , resulting in the exchange of a highly conserved nucleotide. The protease InhA2 of $B$. thuringiensis also shows an aberrant PlcR consensus sequence (Fedhila et al., 2003), and the effect of base exchanges within the PlcR box on regulatordependent activity has been investigated. It was found that mutations within the PlcR consensus lead to lower transcription depending on their location (Gohar et al., 2008). However, the substitution that we identified in the BA1995 PlcR box was not included in the earlier study. Our results showing that BA1995 is still transcribed at a low level in the absence of PlcR imply that changes in the more conserved residues lower the specificity of PlcR for its target sequence, as shown by Gohar et al. (2008). Additionally, the ability of PlcR to recognize an aberrant recognition sequence suggests that the $\mathrm{PlcR}$ regulon is larger than anticipated.

One striking phenotype of bacteria harbouring a functional $\mathrm{PlcR}$ is their haemolytic behaviour on blood agar plates. PlcR-PapR-expressing bacteria were clearly haemolytic, and anthrolysin, the best-characterized haemolysin of $B$. anthracis (Mosser \& Rest, 2006; Shannon et al., 2003), was found to be the main contributor, causing lysis of human erythrocytes in PlcR-positive and -negative bacteria. Although sphingomyelinase is transcribed at a low level, its contribution to haemolysis of human erythrocytes appears to be negligible. However, the content of sphingomyelin in the membrane of human red blood cells is low when compared with other species. In sheep, sphingomyelin can make up to $50 \%$ of the phospholipids present in the red blood cell membrane (Ikezawa et al., 1980); therefore, for detection of sphingomyelinase activity, erythrocytes of other species are expected to be a better indicator.

Macrophages are key players during anthrax pathogenesis, functioning as vehicles for transport of phagocytosed 
(a)

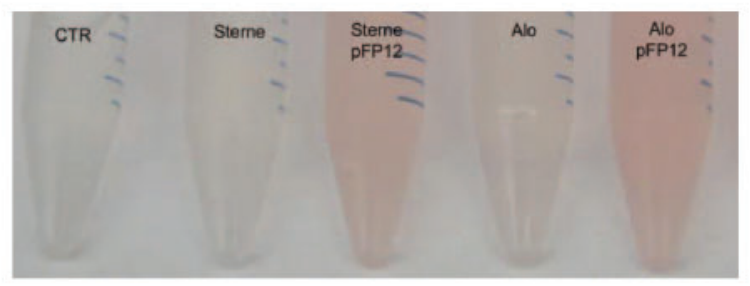

(b)

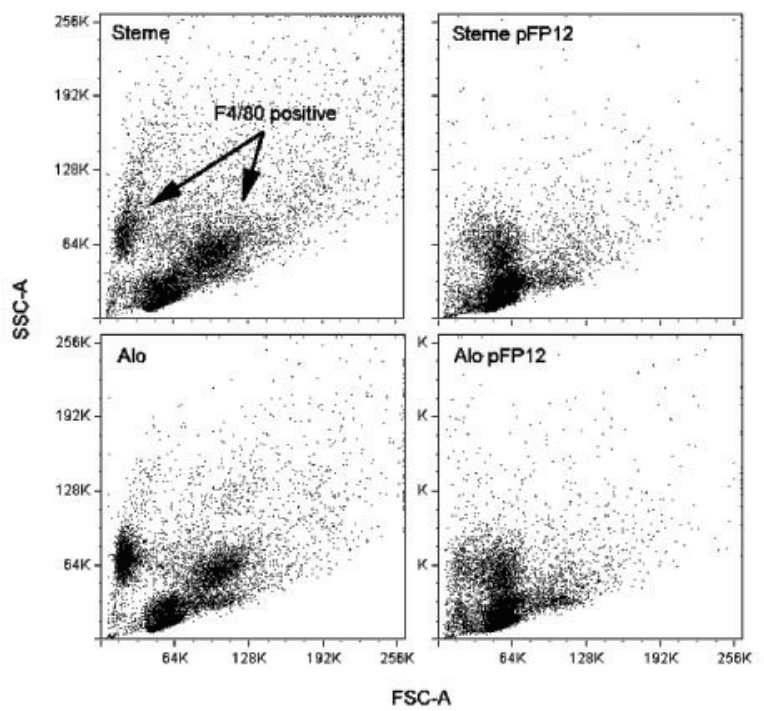

(c)

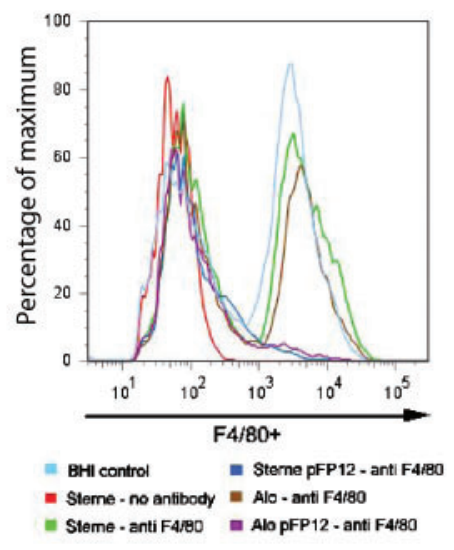

(d)

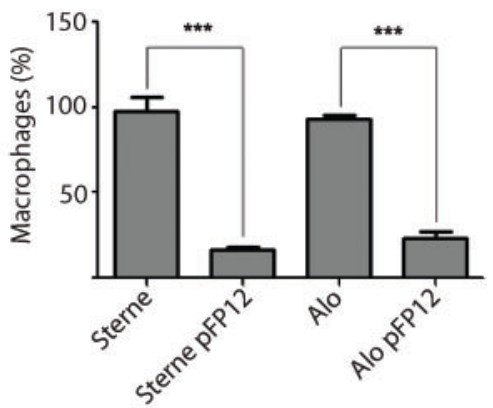

Fig. 6. In vivo macrophage toxicity of proteins secreted by Bacillus. (a) Intraperitoneal (IP) washes of mice $(n=2)$ treated with $500 \mu \mathrm{l}$ filter-sterilized bacterial culture supernatants derived from $B$. anthracis or $B$. anthracis anthrolysin knockout strain in the presence or absence of PlcR-PapR. Control animals were treated with BHI broth alone. (b) Flow cytometry analysis showing forward and side scatter of IP washes derived from mice treated with cell-free bacterial culture supernatants. Arrows indicate macrophage $(\mathrm{F} 4 / 80+)$ populations lacking in washes treated with supernatants derived from PlcR-PapR-expressing bacteria. (c) Chart of macrophage $(\mathrm{F} 4 / 80+)$ populations present in IP washes of mice treated with bacterial supernatants or with BHI alone. (d) Quantification of macrophages (\%) present in the IP washes of mice treated with bacterial culture supernatants. Percentages represent the number of $\mathrm{F} 4 / 80$ + cells relative to the entire cell population present in the IP wash. Shown are mean \pm SD from one representative experiment out of three.

spores to peripheral lymph nodes. Mosser \& Rest (2006) have shown that recombinant anthrolysin and supernatants derived from B. anthracis are able to kill a variety of cells, including macrophages derived from peripheral human blood. Surprisingly, we found no toxic response in macrophages treated with supernatants from $B$. anthracis Sterne. However, the final concentration of $25 \%$ supernatant in our cell assay may be lower than the concentrations used by Mosser \& Rest (2006). In contrast, macrophages treated in vitro and in vivo with culture supernatants derived from PlcR-PapR-expressing bacteria were killed, and deletion of anthrolysin expression did not have an effect, indicating that toxin(s) other than anthrolysin were responsible for the killing. For B. cereus, the PlcR-dependent expression of multiple toxins has been described, including the nonhaemolytic enterotoxin (Nhe), which is a three-component toxin associated with food poisoning (Lund \& Granum, 1996). B. anthracis is known to express NheA in a
PlcR-independent manner (Mendelson et al., 2004), yet it is likely that activation of PlcR increases expression, since its promoter harbours a PlcR box. Concomitantly, we found NheA (BA1887) in the supernatant of B. anthracis expressing PlcR. Many other toxins and virulence factors, including proteases and phospholipases, are dependent on PlcR (Gohar et al., 2002), and all of them could contribute to toxicity towards macrophages. The specificity with which the macrophage population was targeted in our in vivo studies was surprising, indicating a crucial role for these cells. PlcR is truncated in all known $B$. anthracis strains to date, and silencing of these unknown toxin(s) that mediate macrophage killing could be the reason why mutation of $\mathrm{PlcR}$ was favoured in these strains. The exact mechanism by which PlcR-reconstituted $B$. anthracis attacks macrophages and the identity of the toxin(s) involved deserve further investigation, especially in strains harbouring both virulence plasmids. 


\section{ACKNOWLEDGEMENTS}

This work was supported by the Intramural Research Program of the National Institutes of Health (NIH), National Institute of Allergy and Infectious Diseases (NIAID), Bethesda, MD, USA. We thank Zachary Newman for supplying BMDM cells, Devorah Crown for assistance with animal experiments, the NIAID RTB for performing 2D SDSPAGE and MS, and Dr Clinton Leysath for assistance with flow cytometry. The views expressed in this article are those of the authors and do not necessarily reflect the official policy or position of the Department of the Navy, Department of Defense, nor the US Government.

\section{REFERENCES}

Agaisse, H., Gominet, M., Okstad, O. A., Kolstø, A. B. \& Lereclus, D. (1999). PlcR is a pleiotropic regulator of extracellular virulence factor gene expression in Bacillus thuringiensis. Mol Microbiol 32, 10431053.

Beecher, D. J. \& Wong, A. C. (2000). Cooperative, synergistic and antagonistic haemolytic interactions between haemolysin BL, phosphatidylcholine phospholipase $\mathrm{C}$ and sphingomyelinase from Bacillus cereus. Microbiology 146, 3033-3039.

Bouillaut, L., Perchat, S., Arold, S., Zorrilla, S., Slamti, L., Henry, C., Gohar, M., Declerck, N. \& Lereclus, D. (2008). Molecular basis for group-specific activation of the virulence regulator PlcR by PapR heptapeptides. Nucleic Acids Res 36, 3791-3801.

Burdon, K. L. (1956). Useful criteria for the identification of Bacillus anthracis and related species. J Bacteriol 71, 25-42.

Chitlaru, T., Gat, O., Gozlan, Y., Ariel, N. \& Shafferman, A. (2006). Differential proteomic analysis of the Bacillus anthracis secretome: distinct plasmid and chromosome $\mathrm{CO}_{2}$-dependent cross talk mechanisms modulate extracellular proteolytic activities. J Bacteriol 188, 3551-3571.

Dai, Z. \& Koehler, T. M. (1997). Regulation of anthrax toxin activator gene $(\operatorname{atx} A)$ expression in Bacillus anthracis: temperature, not $\mathrm{CO}_{2} /$ bicarbonate, affects AtxA synthesis. Infect Immun 65, 25762582.

Drobniewski, F. A. (1993). Bacillus cereus and related species. Clin Microbiol Rev 6, 324-338.

Emanuelsson, O., Brunak, S., von Heijne, G. \& Nielsen, H. (2007). Locating proteins in the cell using TargetP, SignalP and related tools. Nat Protoc 2, 953-971.

Fedhila, S., Gohar, M., Slamti, L., Nel, P. \& Lereclus, D. (2003). The Bacillus thuringiensis PlcR-regulated gene inhA2 is necessary, but not sufficient, for virulence. J Bacteriol 185, 2820-2825.

Gardy, J. L., Laird, M. R., Chen, F., Rey, S., Walsh, C. J., Ester, M. \& Brinkman, F. S. (2005). PSORTb v.2.0: expanded prediction of bacterial protein subcellular localization and insights gained from comparative proteome analysis. Bioinformatics 21, 617-623.

Gohar, M., Okstad, O. A., Gilois, N., Sanchis, V., Kolstø, A. B. \& Lereclus, D. (2002). Two-dimensional electrophoresis analysis of the extracellular proteome of Bacillus cereus reveals the importance of the PlcR regulon. Proteomics 2, 784-791.

Gohar, M., Gilois, N., Graveline, R., Garreau, C., Sanchis, V. \& Lereclus, D. (2005). A comparative study of Bacillus cereus, Bacillus thuringiensis and Bacillus anthracis extracellular proteomes. Proteomics 5, 3696-3711.

Gohar, M., Faegri, K., Perchat, S., Ravnum, S., Okstad, O. A., Gominet, M., Kolstø, A. B. \& Lereclus, D. (2008). The PlcR virulence regulon of Bacillus cereus. PLoS ONE 3, e2793.
Helgason, E., Okstad, O. A., Caugant, D. A., Johansen, H. A., Fouet, A., Mock, M., Hegna, I. \& Kolstø, A. B. (2000). Bacillus anthracis, Bacillus cereus, and Bacillus thuringiensis - one species on the basis of genetic evidence. Appl Environ Microbiol 66, 2627-2630.

Hoffmaster, A. R., Ravel, J., Rasko, D. A., Chapman, G. D., Chute, M. D., Marston, C. K., De, B. K., Sacchi, C. T., Fitzgerald, C. \& other authors (2004). Identification of anthrax toxin genes in a Bacillus cereus associated with an illness resembling inhalation anthrax. Proc Natl Acad Sci U S A 101, 8449-8454.

Hoffmaster, A. R., Hill, K. K., Gee, J. E., Marston, C. K., De, B. K., Popovic, T., Sue, D., Wilkins, P. P., Avashia, S. B. \& other authors (2006). Characterization of Bacillus cereus isolates associated with fatal pneumonias: strains are closely related to Bacillus anthracis and harbor B. anthracis virulence genes. J Clin Microbiol 44, 33523360 .

Ikezawa, H., Mori, M. \& Taguchi, R. (1980). Studies on sphingomyelinase of Bacillus cereus: hydrolytic and hemolytic actions on erythrocyte membranes. Arch Biochem Biophys 199, 572-578.

Ivanova, N., Sorokin, A., Anderson, I., Galleron, N., Candelon, B., Kapatral, V., Bhattacharyya, A., Reznik, G., Mikhailova, N. \& other authors (2003). Genome sequence of Bacillus cereus and comparative analysis with Bacillus anthracis. Nature 423, 87-91.

Ivins, B. E., Ezzell, J. W., Jr, Jemski, J., Hedlund, K. W., Ristroph, J. D. \& Leppla, S. H. (1986). Immunization studies with attenuated strains of Bacillus anthracis. Infect Immun 52, 454-458.

Klee, S. R., Ozel, M., Appel, B., Boesch, C., Ellerbrok, H., Jacob, D., Holland, G., Leendertz, F. H., Pauli, G. \& other authors (2006). Characterization of Bacillus anthracis-like bacteria isolated from wild great apes from Côte d'Ivoire and Cameroon. J Bacteriol 188, 53335344.

Klichko, V. I., Miller, J., Wu, A., Popov, S. G. \& Alibek, K. (2003). Anaerobic induction of Bacillus anthracis hemolytic activity. Biochem Biophys Res Commun 303, 855-862.

Kolstø, A. B., Tourasse, N. J. \& Okstad, O. A. (2009). What sets Bacillus anthracis apart from other Bacillus species? Annu Rev Microbiol 63, 451-476.

Leppla, S. H. (1988). Production and purification of anthrax toxin. Methods Enzymol 165, 103-116.

Lereclus, D., Agaisse, H., Gominet, M., Salamitou, S. \& Sanchis, V. (1996). Identification of a Bacillus thuringiensis gene that positively regulates transcription of the phosphatidylinositol-specific phospholipase C gene at the onset of the stationary phase. J Bacteriol 178, 2749-2756.

Lund, T. \& Granum, P. E. (1996). Characterisation of a nonhaemolytic enterotoxin complex from Bacillus cereus isolated after a foodborne outbreak. FEMS Microbiol Lett 141, 151-156.

Makarova, K. S., Aravind, L. \& Koonin, E. V. (1999). A superfamily of archaeal, bacterial, and eukaryotic proteins homologous to animal transglutaminases. Protein Sci 8, 1714-1719.

McAllister, R. D., Singh, Y., Du Bois, W. D., Potter, M., Boehm, T., Meeker, N. D., Fillmore, P. D., Anderson, L. M., Poynter, M. E. \& other authors (2003). Susceptibility to anthrax lethal toxin is controlled by three linked quantitative trait loci. Am J Pathol 163, 1735-1741.

Mendelson, I., Tobery, S., Scorpio, A., Bozue, J., Shafferman, A. \& Friedlander, A. M. (2004). The NheA component of the nonhemolytic enterotoxin of Bacillus cereus is produced by Bacillus anthracis but is not required for virulence. Microb Pathog 37, 149154.

Mignot, T., Mock, M., Robichon, D., Landier, A., Lereclus, D. \& Fouet, A. (2001). The incompatibility between the PlcR- and AtxA-controlled regulons may have selected a nonsense mutation in Bacillus anthracis. Mol Microbiol 42, 1189-1198. 
Mikesell, P., Ivins, B. E., Ristroph, J. D. \& Dreier, T. M. (1983) Evidence for plasmid-mediated toxin production in Bacillus anthracis. Infect Immun 39, 371-376.

Mock, M. \& Fouet, A. (2001). Anthrax. Annu Rev Microbiol 55, 647-671.

Mosser, E. M. \& Rest, R. F. (2006). The Bacillus anthracis cholesteroldependent cytolysin, anthrolysin $\mathrm{O}$, kills human neutrophils, monocytes and macrophages. BMC Microbiol 6, 56.

Okstad, O. A., Gominet, M., Purnelle, B., Rose, M., Lereclus, D. \& Kolstø, A. B. (1999). Sequence analysis of three Bacillus cereus loci carrying PlcR-regulated genes encoding degradative enzymes and enterotoxin. Microbiology 145, 3129-3138.

Park, S. \& Leppla, S. H. (2000). Optimized production and purification of Bacillus anthracis lethal factor. Protein Expr Purif 18, 293-302.

Passalacqua, K. D., Varadarajan, A., Byrd, B. \& Bergman, N. H. (2009). Comparative transcriptional profiling of Bacillus cereus sensu lato strains during growth in $\mathrm{CO}_{2}$-bicarbonate and aerobic atmospheres. PLoS ONE 4, e4904.

Pomerantsev, A. P., Kalnin, K. V., Osorio, M. \& Leppla, S. H. (2003). Phosphatidylcholine-specific phospholipase $\mathrm{C}$ and sphingomyelinase activities in bacteria of the Bacillus cereus group. Infect Immun 71, 6591-6606.

Pomerantsev, A. P., Pomerantseva, O. M. \& Leppla, S. H. (2004). A spontaneous translational fusion of Bacillus cereus PlcR and PapR activates transcription of PlcR-dependent genes in Bacillus anthracis via binding with a specific palindromic sequence. Infect Immun 72, 5814-5823.

Pomerantsev, A. P., Camp, A. \& Leppla, S. H. (2009). A new minimal replicon of Bacillus anthracis plasmid pXO1. J Bacteriol 191, 5134-5146.
Rasko, D. A., Altherr, M. R., Han, C. S. \& Ravel, J. (2005). Genomics of the Bacillus cereus group of organisms. FEMS Microbiol Rev 29, 303329.

Read, T. D., Peterson, S. N., Tourasse, N., Baillie, L. W., Paulsen, I. T., Nelson, K. E., Tettelin, H., Fouts, D. E., Eisen, J. A. \& other authors (2003). The genome sequence of Bacillus anthracis Ames and comparison to closely related bacteria. Nature 423, 81-86.

Reddy, A., Battisti, L. \& Thorne, C. B. (1987). Identification of selftransmissible plasmids in four Bacillus thuringiensis subspecies. J Bacteriol 169, 5263-5270.

Ross, C. L. \& Koehler, T. M. (2006). plcR papR-independent expression of anthrolysin $\mathrm{O}$ by Bacillus anthracis. J Bacteriol 188, 7823-7829.

Sastalla, I., Chim, K., Cheung, G. Y., Pomerantsev, A. P. \& Leppla, S. H. (2009). Codon-optimized fluorescent proteins designed for expression in low-GC Gram-positive bacteria. Appl Environ Microbiol 75, 20992110.

Shannon, J. G., Ross, C. L., Koehler, T. M. \& Rest, R. F. (2003). Characterization of anthrolysin $\mathrm{O}$, the Bacillus anthracis cholesteroldependent cytolysin. Infect Immun 71, 3183-3189.

Slamti, L., Perchat, S., Gominet, M., Vilas-Boas, G., Fouet, A., Mock, M., Sanchis, V., Chaufaux, J., Gohar, M. \& other authors (2004). Distinct mutations in PlcR explain why some strains of the Bacillus cereus group are nonhemolytic. J Bacteriol 186, 3531-3538.

Tweten, R. K. (2005). Cholesterol-dependent cytolysins, a family of versatile pore-forming toxins. Infect Immun 73, 6199-6209.

Edited by: S. J. Foster 